\title{
Flow Diversion versus Traditional Endovascular Coiling Therapy: Design of the Prospective LARGE Aneurysm Randomized Trial
}

\author{
A.S. Turk III, R.H. Martin, D. Fiorella, J. Mocco, A. Siddiqui, and A. Bonafe
}

\begin{abstract}
BACKGROUND AND PURPOSE: The goal of aneurysm treatment is occlusion of an aneurysm without morbidity or mortality. Using well-established, traditional endovascular techniques, this is generally achievable with a high level of safety and efficacy. These techniques involve either constructive treatment of the aneurysm (coils with or without an intravascular stent) or deconstruction (coil occlusion) of the aneurysm and the parent artery. While established as safe and efficacious, the constructive treatment of large and giant aneurysms with coils has typically been associated with relatively lower rates of complete occlusion and higher rates of recurrence. Parent artery deconstruction, though immediately efficacious in achieving complete and durable occlusion, does require occlusion of a major intracranial blood vessel and is associated with risk of stroke.
\end{abstract}

MATERIALS AND METHODS: Flow diversion represents a new technology that can be used to constructively treat large and giant aneurysms. Once excluded successfully, the vessel reconstruction and aneurysm occlusion appears durable. The ability to definitively reconstruct cerebral blood vessels is an attractive approach to these large and giant complex aneurysms and allows the treatment of some aneurysms which were previously not amenable to other therapies. By comparison, conventional coiling techniques have traditionally been used for endovascular treatment of large aneurysms. Large and giant aneurysms that are amenable to either flow diversion or traditional endovascular treatment will be randomized to either therapy with FDA (or appropriate regulatory body) approved devices.

RESULTS: The trial is currently enrolling and results of the data are pending the completion of enrollment and follow-up.

CONCLUSIONS: This paper details the trial design of the LARGE trial, a blinded, prospective randomized trial of large anterior circulation aneurysms amenable to either traditional endovascular treatments using coils or reconstruction with flow diverters.

ABBREVIATIONS: IA = intracranial aneurysms; LARGE = Large Aneurysm Randomized Trial: Flow Diversion Versus Traditional GDC Based Endovascular Therapy

$\mathbf{C}_{\mathrm{r}}^{\mathrm{c}}$ erebral aneurysms (ie, intracranial aneurysms [IAs]) are a relatively common cerebrovascular abnormality that has been reported to occur in $0.8 \%-10.0 \%$ of the population. ${ }^{1-4}$ The risk of IA rupture has been shown to increase with increasing size. ${ }^{5,6}$ The most common presentation of IAs is subarachnoid hemorrhage, the annual incidence of which varies by geographic

Received August 29, 2013; accepted after revision November 4.

From the Department of Radiology (A.S.T., R.H.M.), Medical University of South Carolina, Charleston, South Carolina; Department of Neurological Surgery (D.F.), Stony Brook Medicine, Stony Brook, New York; Department of Neurological Surgery (I.M.) Vanderbilt University Medical Center, Nashville, Tennessee; Department of Neurosurgery (A.S.), University of Buffalo, Buffalo, New York; and Department of Neurosurgery (A.B.), Hospital Gui de Chauliac, Centre Hospitalier Universitaire de Montpellier, Montpellier, France.

This research was supported by Codman, Microvention, Penumbra, and Stryker.

Please address correspondence to Aquilla S. Turk, III, DO, Professor, Department of Radiology, Medical University of South Carolina, 96 Jonathan Lucas St, MSC 323 , Charleston, SC 29425; e-mail: turk@musc.edu

三 Indicates article with supplemental on-line table

http://dx.doi.org/10.3174/ajnr.A3968 region from 10 to 20 per 100,000 with a case-fatality rate of $51 \%{ }^{7,8}$ For these reasons, most physicians recommend treatment for large ( $>10 \mathrm{~mm})$, intradural IAs.

Historically, the treatment of very large and giant aneurysms has focused on deconstructive approaches in which the parent artery bearing the aneurysm is occluded, or complex microsurgical procedures requiring flow arrest with clip reconstruction or entailing bypass strategies to distal-downstream cerebral circulation. ${ }^{9-11}$ This requires that a patient has ample collateral channels to compensate for the occlusion of the artery supplying the aneurysm and typically this must be confirmed by a test balloon occlusion. ${ }^{12,13}$ This method of aneurysm treatment yields an immediate and durable cure of the lesion treated and has been shown to have an acceptable safety profile with morbidity and mortality rates ranging from $0 \%-16 \%$. These rates appear to be dependent upon rigorous physiologic assessment of collateral circulation reserve using intraprocedural hypotensive challenges and or postprocedural imaging with CBF assessment using SPECT or other modalities. When parent 
vessel deconstruction is used for the treatment of symptomatic aneurysms, the presenting clinical symptoms have been reported to resolve in $75 \%$ of cases, improve in $10 \%$, and remain unchanged in $15 \%$ of cases. ${ }^{9}$ When feasible, deconstructive treatment remains a viable treatment strategy for these lesions.

Currently, the most common endovascular treatment approach to IAs has been constructive endosaccular coil embolization. Despite the popularity of coil embolization for the treatment of IAs, incomplete occlusion of the target IA is surprisingly common, approaching $65 \%$ in aneurysms larger than $10 \mathrm{~mm} .{ }^{14-16}$ Several factors are known to predict the likelihood of complete IA occlusion after constructive treatment with coil embolization. The most important factors predicting incomplete occlusion are overall lesion diameter and neck size. Large and giant IAs and those with wide necks are even less likely to have complete occlusion after coil embolization. ${ }^{16,17}$ The presence of intraluminal thrombus is also highly associated with repeated incidences of recurrence and retreatment. Other accepted morphologic predictors of incomplete IA occlusion include aneurysm shape and location. Complete or near complete IA occlusion is the goal of endosaccular aneurysm treatment. Several studies have shown that incomplete occlusion of the target IA is a risk factor for subsequent IA regrowth and retreatment and it is believed that incompletely occluded aneurysms retain their risk for rupture and subarachnoid hemorrhage. ${ }^{18}$ The evolution of coil technology and the advent of adjunctive devices such as stents and balloons have greatly facilitated the constructive treatment of large and giant, wide-neck IAs. With modern constructive techniques, peri-procedure morbidity rates for the treatment of these challenging aneurysms has declined from $25 \%$ to $2 \%-11 \% .{ }^{19}$ Moreover, there are some data to suggest that the application of these adjunctive devices, particularly endoluminal stents, may improve the rates of complete aneurysm occlusion and support the durability of treatment. ${ }^{20}$

Endoluminal aneurysm reconstruction using flow diverters represents a new endovascular approach to IA treatment. Emerging clinical data have shown that this approach may yield considerably higher rates of complete aneurysm occlusion in comparison with traditional endosaccular approaches. ${ }^{21}$ The prospective, multicenter, Pipeline for Uncoilable or Failed Aneurysms (PUFS) study that trialed the treatment of large and giant wide-neck carotid aneurysms with the Pipeline embolization device (Covidien, Irvine, California) flow diverter reported complete angiographic occlusion rates approaching $90 \%$ at 1 -year follow-up. ${ }^{22}$

The recent PUFS data reported a $15 \%$ major neurologic adverse event rate and $44 \%$ minor adverse event rate with flow diverters. Particularly of concern are unexplained incidences of catastrophic delayed spontaneous ipsilateral intracranial parenchymal hemorrhage and delayed aneurysmal ruptures that have been reported in up to $5 \%$ of cases after flow diversion and have not been typically associated with standard endosaccular coil embolization or parent artery deconstruction. ${ }^{23-25}$

To date, no study has directly compared the safety and efficacy of flow diverters with conventional endovascular coil-based techniques for the treatment of large and giant, wide-neck IAs that are amenable to either treatment approach. In this article, we describe the design and methods of a large, ongoing randomized clinical trial (NCT01762137) to assess efficacy and safety of traditional endovascular therapy using coils with or without adjunctive de- vices in a reconstructive or deconstructive manner versus approved flow-diversion technologies in the treatment of large anterior circulation intracranial aneurysms.

\section{METHODS AND DESIGN \\ Design}

LARGE is an international prospective multicenter trial to compare the safety and efficacy of flow diversion to traditional endovascular techniques for the treatment of anterior circulation large and giant aneurysms. Subjects will be randomized to either flow diversion or endovascular coiling (reconstructive coiling with or without adjunctive devices [stents, balloons] or deconstruction) cohorts in a 1:1 fashion and will be assessed for the primary outcome at 6 months with subsequent outcomes until 3 years from aneurysm repair. The primary objective is to show that flow diversion is noninferior to endovascular coiling at 180 days from aneurysm treatment on the primary end point by less than an absolute difference of $15 \%$. The primary outcome is a combined efficacy and safety end point defined by greater than $90 \%$ angiographic occlusion with stable or decreased aneurysm size on cross-sectional imaging (CT or MR) at 180 days postprocedure and freedom from any major neurologic event (defined as change in NIHSS from baseline $>4$ points) or death at 180 days postprocedure.

\section{Patient Population}

The On-line Table shows patient eligibility criteria. Figure 1 shows the flow chart of patients through LARGE. The LARGE trial includes current flow-diversion on-label patients, with aneurysms larger than $1 \mathrm{~cm}$ located on the internal carotid artery below the level of the posterior communicating artery. The aneurysm must be amenable to either conventional endovascular therapy or flow diversion according to the operator's discretion. Many patients with large paraclinoid aneurysms present clinically with visual or ocular findings. If the patient's presentation includes any eye signs or symptoms, the patient will be referred for evaluation by a neuro-ophthalmologist preprocedurally.

\section{Randomization}

Randomization will occur in a 1:1 ratio to either flow diversion or endovascular coiling. The covariate adaptive randomization balances treatment assignment based on aneurysm location (intradural versus extradural), presence of intraluminal thrombus ("yes" versus "no"), aneurysm shape (saccular versus fusiform), prior balloon test occlusion ("yes" versus "no"), and current status of treatment groups within and across clinical sites. Once the patient is determined to meet all study eligibility criteria, covariate adaptive randomization takes place centrally via the LARGE Study Web site on the WebDCU (https://webdcu.musc.edu).

\section{Treatment}

Subjects assigned to coil embolization will undergo treatment of the target IA with endovascular coiling with FDA-approved technologies. Procedures will be performed according to the technology instructions for use. The goal of coil treatment is to completely occlude the IA. Other devices (eg, intravascular balloons ["balloon remodeling"], intravascular stents, dual catheters, etc) may be used adjunctively to deliver or direct coils into the target IA. Alternatively, deconstructive techniques with parent vessel occlusion utilizing en- 


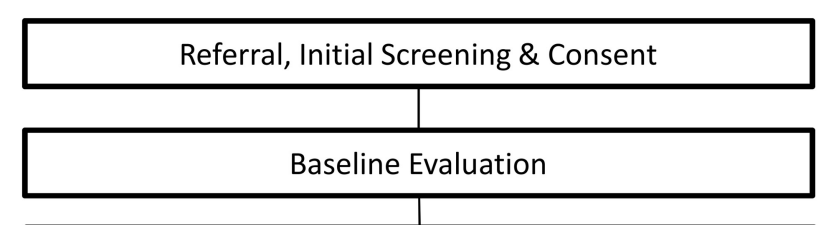

No more than 30 days may lapse between baseline assessment and the study procedure

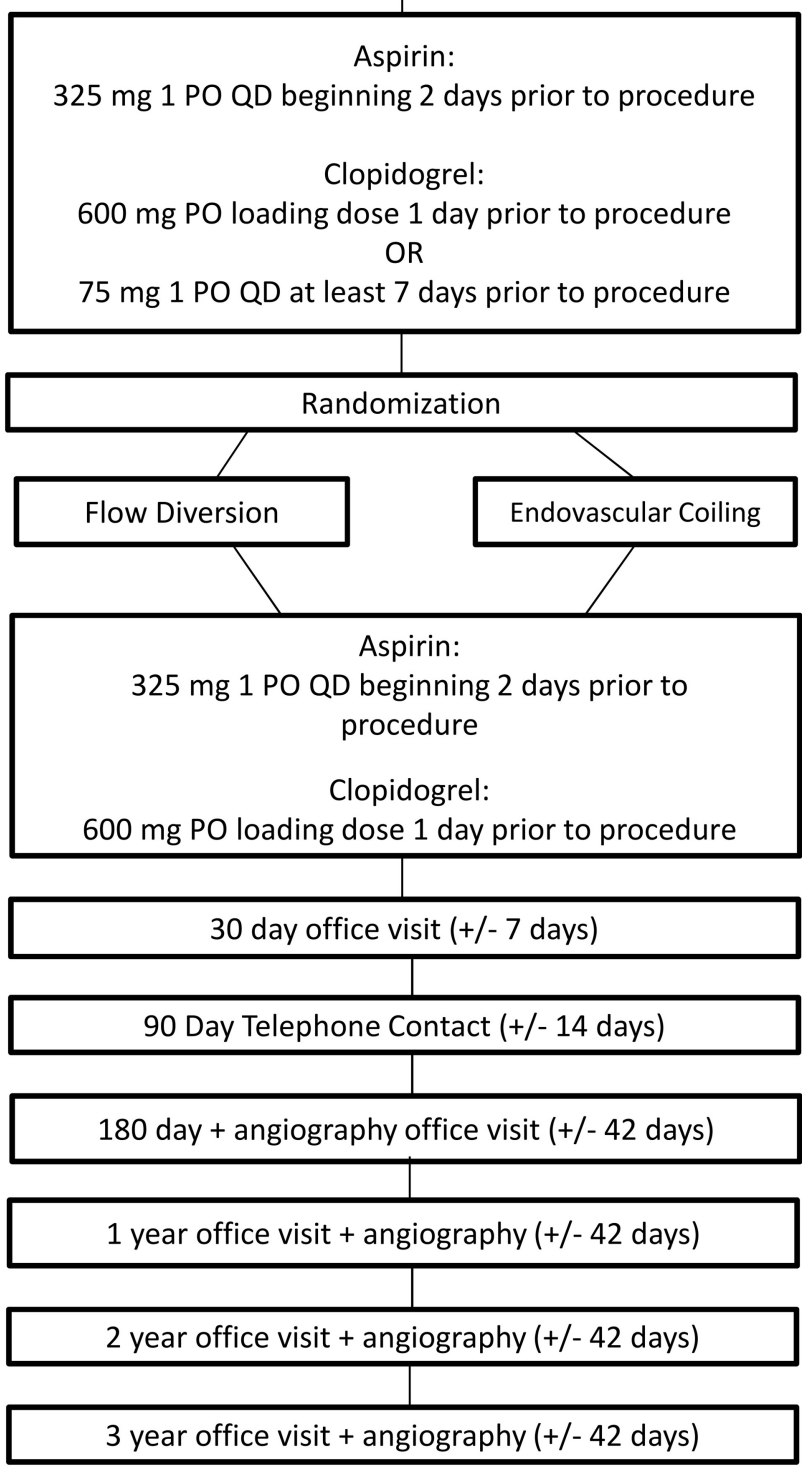

FIG 1. Study flow from referral through follow-up.

dovascular coiling are allowed if this option is felt to be the best treatment technique for the patient. The patient must first successfully pass a balloon test occlusion before vessel occlusion.

Subjects assigned to flow diversion will undergo placement of flow diverter(s) across the target IA. The placement procedure is described briefly in Fig 1 and in more detail in the device instructions for use document. One or more flow diverters may be placed as deemed necessary by the investigator.

Aspirin and clopidrogel are used before flow diverter placement or endovascular coiling embolization. Aspirin is used for at least 1 year after endovascular coiling or flow diverter placement.
Clopidogrel is used for at least 3 months after endovascular coiling embolization or flow diverter placement. Aspirin and/or clopidogrel may be used beyond (or at higher doses) than the indicated regimen, if appropriate clinically (eg, patient previously taking aspirin for coronary artery disease prophylaxis).

\section{Primary Outcome}

The primary outcome is a dual end point of efficacy and safety. Efficacy is defined as $>90 \%$ angiographic occlusion with stable (or decreased) aneurysm size on cross-sectional imaging (CT or MR) at 180 days postprocedure. Safety is defined as the patient being free of any major ipsilateral neurologic event (defined as change in NIHSS from baseline $>4$ points) including ipsilateral neurologic stroke or death at 180 days postprocedure.

\section{Data Safety Monitoring Board}

A data safety monitoring board will comprise 4 members not participating in the trial and will include a neuroradiologist, neurologist, neurosurgeon, and statistician. The data safety monitoring board will exercise review of the overall safety of the trial, periodically review all adverse events occurring in the trial, and make recommendations to adjustments in the study protocol, should any be considered necessary for safety or other related reasons.

\section{Sample Size}

The sample size of 316 randomized subjects was selected. Sample size was based on the noninferiority design whereby the proportion of success under the endovascular coiling arm (the active control arm) is considered to be 0.75 , the noninferiority margin $(\Delta)$ is set at 0.15 , the type I error is selected to be 0.025 , there are 2 interim analyses for futility, a $15 \%$ inflation because of potential loss to follow-up, and $80 \%$ power.

\section{Statistical Analyses}

Statistical analyses are based on a noninferiority trial to test the hypothesis that the efficacy of flow diversion is not worse than that of endovascular coiling by more than a prespecified absolute amount $\delta=15 \%$ (ie, the noninferiority margin or prespecified clinically unimportant difference) for the treatment of large and giant aneurysms. Therefore, rejection of the null hypothesis indicates that the flow diversion is not inferior to the endovascular coiling by this prespecified amount. The primary analysis will be intent-to-treat and will assess efficacy with respect to the proportion of subjects with successful outcome at 180 days postrandomization using a generalized linear model adjusting for baseline aneurysm location (intradural versus extradural), presence of intraluminal thrombus ("yes" versus "no"), aneurysm shape (saccular versus fusiform), and undergoing balloon test occlusion ("yes" versus "no"). The primary approach to handling missing primary outcome data, ie, if a subject has a missing angiogram at the 180-day visit or does not attend the 180-day clinical follow-up visit, will be to consider the subject a treatment failure for the primary effectiveness end point.

Additional potentially confounding variables (ie, sex, race, ethnicity, baseline risk factors) will be considered as covariates in secondary analyses of the primary outcome. Univariate analyses 
of these covariates will first be conducted to determine inclusion in the multivariate model.

As specified in the objectives, if noninferiority is demonstrated, then superiority of the safety end point will be assessed. Safety outcomes include the proportion of subjects who experience any treatment-related serious adverse events during the treatment phase and up to 180 days following completion of the treatment. The treatment-related serious adverse events will be considered along with the following:

1. Neurologic deterioration during the hospitalization phase.

2. All deaths by cause (broad categories) within 180 days of randomization.

3. Incidence of neurologic death by 180 days.

A number of secondary analyses will be conducted:

- Incidence of device or procedure related adverse events at 180 days, 1 year, and 3 years.

- Aneurysm rupture or retreatment of index aneurysm rates at 180 days, 1 year, and 3 years.

- Change in clinical functional outcome at 180 days, 1 year, and 3 years postendovascular treatment procedure, as measured by an increase in the modified Rankin Scale from baseline.

- Incidence of worsening of baseline neurologic signs/symptoms as measured by NIHSS or ophthalmologic examination related to target IA at 180 days.

- Number of inpatient hospital (and re-hospitalized) days (subgrouped $>7$ days) at 180 days, 1 year, and 3 years.

- Packing attenuation as measured by volumetric filling of the aneurysm if aneurysm is coiled.

- Device cost of therapy at treatment and any subsequent retreatment.

- Procedure time, as measured as the time from placement of the treating guide catheter for purposes of aneurysm treatment (not balloon test occlusion) until guide catheter removal.

Prespecified subgroup analyses will also be conducted on clinical and angiographic outcomes for the following:

- Subjects with aneurysms $10-20 \mathrm{~mm}$ and $>2 \mathrm{~cm}$.

- Intradural versus extradural location.

- Reconstructive versus deconstructive technique.

- Downstream flow-related ischemic stroke, parenchymal hemorrhage, subarachnoid hemorrhage.

- Complete aneurysm occlusion and no neurologic events at 6 months.

- IA neck size $\geq 4 \mathrm{~mm}$ versus $<4 \mathrm{~mm}$.

- Current/former smoker versus never smoker.

Technical success, defined as: for flow diversion, the proportion of patients in whom at least 1 attempt was made to pass the access catheter distal to the target IA in whom the final location of placed flow diverters covers the IA neck. For endovascular coiling, the proportion of patients in whom at least 1 attempt was made to pass the access catheter into the target IA fundus (for coil delivery) in whom at least 1 coil was left behind in the target IA. If the plan is for deconstructive treatment, then the parent vessel supplying the artery is occluded without residual flow.

All models will be assessed with and without covariates (age, an- eurysm location, etc); this is in keeping with our randomization scheme and is not anticipated to negatively affect the power of the test.

Two protocol-specified interim analyses for futility are planned to be conducted when approximately one-third ( $n=$ $105)$ and two-thirds $(n=210)$ of the total required number of randomized subjects have been evaluated for the primary outcome. These interim analyses will use the error spending function method with O'Brien and Fleming-type stopping guidelines. ${ }^{26-28}$ The error spending function distributes the type I and II error rates across the interim monitoring points giving the flexibility of changing the intervals of monitoring while still preserving the overall type I and II error rates. The O'Brien and Fleming-type boundary is considered conservative as its boundaries make it difficult to terminate a study early on by requiring extreme early evidence of futility. It spends smaller amounts of alpha at the first look and gradually increases the spending as more information is acquired. The trial may be stopped for futility at the planned interim analyses if the test statistic crosses the respective boundaries.

\section{Study Organization and Funding}

The trial was funded in November 2012 through a collaborative sponsorship with equal participation from Codman, Microvention-Terumo, Penumbra, and Stryker. Enrollment began in March 2013 and is currently enrolling patients. The trial is international with sites in the United States, Canada, France, Italy, Spain, and Turkey. The clinical and statistical and data coordination for the trial is being conducted at the Medical University of South Carolina in Charleston, SC.

\section{CONCLUSIONS}

The LARGE trial is an international prospective, randomized multicenter trial designed to compare the safety and efficacy of conventional endovascular techniques versus flow diversion for the treatment of large and giant aneurysms of the carotid siphon that are amenable to either treatment strategy. The primary outcome is a dual end point of efficacy and safety defined as $>90 \%$ angiographic occlusion with stable (or decreased) aneurysm size on cross-sectional imaging (CT or MR) at 180 days postprocedure and freedom of any major ipsilateral neurologic event (defined as change in NIHSS from baseline $>4$ points) including ipsilateral neurologic stroke or death at 180 days postprocedure. Secondary aims follow the primary and secondary outcomes for 3 years. The prespecified subgroup analyses will be performed on aneurysms $10-20 \mathrm{~mm}$ and $>2 \mathrm{~cm}$, intradural versus extradural location, reconstructive versus deconstructive technique, downstream flowrelated ischemic stroke, parenchymal hemorrhage, subarachnoid hemorrhage, complete aneurysm occlusion, no neurologic events at 6 months, and aneurysm neck size $\geq 4 \mathrm{~mm}$ versus $<4 \mathrm{~mm}$. Secondary outcomes will evaluate complications such as downstream flow-related ischemic stroke, parenchymal hemorrhage, subarachnoid hemorrhage, aneurysm rupture, or retreatment of index aneurysm rates; change in clinical functional outcome; incidence of worsening of baseline neurologic signs/ symptoms as measured by NIHSS or ophthalmologic examination; number of inpatient hospital days; packing attenuation as measured by volumetric filling of the aneurysm if coiled; device 
cost of therapy at treatment and any subsequent retreatment; and procedure time. The LARGE trial was designed to enroll 316 patients and have an $80 \%$ power to demonstrate that flow diversion is not inferior to conventional endovascular coiling techniques.

Disclosures: Aquilla Turk—RELATED: Grant: Penumbra, ${ }^{*}$ Covidien, ${ }^{\star}$ MicroVention, ${ }^{*}$ Stryker, ${ }^{\star}$ Siemens, ${ }^{*}$ Codman*; Consulting Fee or Honorarium: Penumbra, Covidien, MicroVention, Stryker, Siemens, Codman; Support for Travel to Meetings for the Study or Other Purposes: Penumbra, MicroVention, Stryker; Provision of Writing Assistance, Medicines, Equipment, or Administrative Support: Penumbra, ${ }^{*}$ Covidien,* MicroVention,* Stryker,* Siemens, ${ }^{*}$ Codman*; UNRELATED: Consultancy: Penumbra, Covidien, MicroVention, Stryker, Siemens, Codman; Grants/Grants Pending: Penumbra, ${ }^{*}$ Covidien, ${ }^{*}$ MicroVention, ${ }^{*}$ Stryker, ${ }^{*}$ Siemens, ${ }^{*}$ Codman; Payment for Lectures (including service on speakers bureaus): Penumbra, Covidien, MicroVention, Stryker, Siemens, Codman; Travel/Accommodations/Meeting Expenses Unrelated to Activities Listed: Penumbra, Covidien, MicroVention, Stryker, Siemens, Codman. Renee Martin—RELATED: Other: Penumbra, ${ }^{*}$ Comments: The Data Coordination Unit (DCU) at the Medical University of South Carolina is receiving funding to support the data management and statistical activities for the LARGE study. As part of the DCU, I am funded for a percentage of my time to participate as the Primary Statistician for the trial. David Fiorella—RELATED: Grant: LARGE Trial,* Comments: Grant from Medical University of South Carolina; Consulting Fee or Honorarium: Codman/JnJ, Covidien/ev3, Comments: Consulting/proctoring; UNRELATED: Grants/Grants Pending: Stenting versus Agressive Medical Therapy for Intracranial Arterial Stenosis (NIH), ${ }^{*}$ LARGE, ${ }^{*}$ Perfusion Imaging Selection of Ischemic Stroke Patients for Endovascular Therapy, ${ }^{*}$ MicroVention, ${ }^{*}$ Low-profile Visualized Intraluminal Support Post Market Approval, ${ }^{*}$ Siemens*; Patents (planned, pending or issued): Codman/JnJ; Royalties: Codman/J\&J. J Mocco—UNRELATED: Consultancy: NFocus, Lazarus Effect, Reverse Medical Pulsar. Adnan Siddiqui-UNRELATED: Board Membership: Codman and Shurtleff, Covidien; Comments: Advisory boards; Consultancy: Codman and Shurtleff, Concentric Medical, Covidien Vascular Therapies, Guidepoint Global Consulting, Penumbra, Stryker, Pulsar Vascular, Microvention; Grants/Grants Pending: The National Institutes of Health (co-investigator: National Institute of Neurological Disorders and Stroke IR01NS064592-01A1; Hemodynamic Induction of Pathologic Remodeling Leading to Intracranial Aneurysms), University at Buffalo (Research Development Award), The National Institutes of Health (co-investigator: National Institutes of Neurosciences and Hospital 5 R01 EB002873-07, Micro-Radiographic Image for Neurovascular Interventions); Payment for Lectures (including service on speakers bureaus): Codman and Shurtleff; Stock/ Stock Options: Hotspur, Intratech Medical, StimSox, Valor Medical, Blockade Medical; Other: Abbott Vascular, for training physicians in endovascular stenting for aneurysms; American Association of Neurological Surgeons' Courses; Penumbra, Comments: Honoraria; OTHER RELATIONSHIPS: Serve on National Steering Committees for: Penumbra. 3D Separator Trail and Covidien Solitaire FR as Primary Treatment for Acute Ischemic Stroke Trial. Alain Bonafe-UNRELATED: Consultancy: Stryker, MicroVention. ${ }^{*}$ money paid to institution.

\section{REFERENCES}

1. Caranci F, Briganti F, Cirillo L, et al. Epidemiology and genetics of intracranial aneurysms. Eur J Radiol. 2013;82:1598-605

2. Molyneux A, Kerr R, Stratton I, et al. International subarachnoid aneurysm trial (ISAT) of neurosurgical clipping versus endovascular coiling in 2143 patients with ruptured intracranial aneurysms: a randomized trial. Lancet 2002;360:1267-74

3. Molyneux AJ, Kerr RS, Yu LM, et al. International subarachnoid aneurysm trial (ISAT) of neurosurgical clipping versus endovascular coiling in 2143 patients with ruptured intracranial aneurysms: a randomized comparison of effects on survival, dependency, seizures, rebleeding, subgroups, and aneurysm occlusion. Lancet 2005;366:809-17

4. FDA. Summary FE. P100018: Chestnut medical Pipeline embolization device. In: Administration FaD, ed. FDA, Washington, DC: Executive Summary; 2011:72

5. International Study of Unruptured Intracranial Aneurysms Investigators. Unruptured intracranial aneurysms - risk of rupture and risks of surgical intervention. $N$ Engl J Med 1998;339:1725-33

6. Gonzalez N, Sedrak M, Martin N, et al. Impact of anatomic features in the endovascular embolization of 181 anterior communicating artery aneurysms. Stroke 2008;39:2776-82

7. Byrne JV, Sohn MJ, Molyneux AJ, et al. Five-year experience in using coil embolization for ruptured intracranial aneurysms: outcomes and incidence of late rebleeding. J Neurosurg 1999;90:656-63

8. Hayakawa M, Murayama Y, Duckwiler GR, et al. Natural history of the neck remnant of a cerebral aneurysm treated with the Guglielmi detachable coil system. J Neurosurg 2000;93:561-68

9. Clarencon F, Bonneville F, Boch AL, et al. Parent artery occlusion is not obsolete in giant aneurysms of the ICA. Experience with verylong-term follow-up. Neuroradiology 2011;53:973-82

10. Higashida RT, Halbach VV, Dowd C, et al. Endovascular detachable balloon embolization therapy of cavernous carotid artery aneurysms: results in 87 cases. J Neurosurg 1990;72:857-63

11. Linskey ME, Sekhar LN, Hirsch W, Jr, et al. Aneurysms of the intracavernous carotid artery: clinical presentation, radiographic features, and pathogenesis. Neurosurgery 1990;26:71-79

12. Larson JJ, Tew JM, Jr, Tomsick TA, et al. Treatment of aneurysms of the internal carotid artery by intravascular balloon occlusion: longterm follow-up of 58 patients. Neurosurgery 1995;36:26-30

13. Wong GK, Poon WS, Chun H, et al. Balloon test occlusion with hypotensive challenge for main trunk occlusion of internal carotid artery aneurysms and pseudoaneurysms. Br J Neurosurg 2010;24:648-52

14. Gallas S, Pasco A, Cottier JP, et al. A multicenter study of 705 ruptured intracranial aneurysms treated with Guglielmi detachable coils. AJNR Am J Neuroradiol 2005;26:1723-31

15. Murayama Y, Nien YL, Duckwiler G, et al. Guglielmi detachable coil embolization of cerebral aneurysms: 11 years' experience. J Neurosurg 2003;98:959-66

16. Vinuela F, Duckwiler G, Mawad M. Guglielmi detachable coil embolization of acute intracranial aneurysm: perioperative anatomical and clinical outcome in 403 patients. J Neurosurg 1997;86:475-82

17. Fernandez Zubillaga A, Guglielmi G, Vinuela F, et al. Endovascular occlusion of intracranial aneurysms with electrically detachable coils: correlation of aneurysm neck size and treatment results. AJNR Am J Neuroradiol 1994;15:815-20

18. Raymond J, Guilbert F, Weill A, et al. Long-term angiographic recurrences after selective endovascular treatment of aneurysms with detachable coils. Stroke 2003;34:1398-403

19. Ortiz R, Song J, Niimi Y, et al. Rate of recanalization and safety of endovascular embolization of intracranial saccular aneurysms framed with GDC 360 coils. Interv Neuroradiol 2008;14:397-401

20. Fiorella D, Hsu D, Woo HH, et al. Very late thrombosis of a Pipeline embolization device construct: case report. Neurosurgery 2010; onsE313-314; discussion onsE314

21. Fiorella D, Lylyk P, Szikora I, et al. Curative cerebrovascular reconstruction with the Pipeline embolization device: the emergence of definitive endovascular therapy for intracranial aneurysms. J Neurointerv Surg 2009;1:56-65

22. Becske T, Kallmes DF, Saatci I, et al. Pipeline for uncoilable or failed aneurysms: results from a multicenter clinical trial. Radiology 2013;267:858-68

23. Kawanabe Y, Sadato A, Taki W, et al. Endovascular occlusion of intracranial aneurysms with Guglielmi detachable coils: correlation between coil packing density and coil compaction. Acta Neurochir (Wien) 2001;143:451-55

24. Reul J, Spetzger U, Weis J, et al. Endovascular occlusion of experimental aneurysms with detachable coils: influence of packing density and perioperative anticoagulation. Neurosurgery 1997;41:116065; discussion 1165-68

25. Tamatani S, Ito Y, Abe H, et al. Evaluation of the stability of aneurysms after embolization using detachable coils: correlation between stability of aneurysms and embolized volume of aneurysms. AJNR Am J Neuroradiol 2002;23:762-67

26. O'Brien PC, Fleming TR. A multiple testing procedure for clinical trials. Biometrics 1979;35:549-56

27. Pampallona STA, Kim KM. Interim monitoring of group sequential trials using spending functions for the Type I and Type II error probabilities. Drug Inf J 2001;35:1113-21

28. Pocock SJ. Statistical and ethical issues in monitoring clinical trials. Stat Med 1993;12:1459-69; discussion 1471-55 